\title{
PRIORIZAÇÃO DE NÓS DE UMA REDE DE SENSORES SEM FIO COM BASE NA ANÁLISE DE DADOS DE REDES SOCIAIS
}

\author{
$\underline{\text { Daniel C. Andrade }}^{1}$; Daniel G. Costa ${ }^{2}$ e João B. Rocha-Junior ${ }^{3}$ \\ 1. Bolsista PROBIC/UEFS, Graduando em Engenharia de Computação, Universidade Estadual de Feira de Santana, \\ e-mail: dca650@gmail.com \\ 2. Orientador, Departamento de Tecnologia, Universidade Estadual de Feira de Santana, \\ e-mail: $\underline{\text { danielgcosta@uefs.br }}$ \\ 3. Colaborador do projeto, Departamento de Ciências Exatas, Universidade Estadual de Feira de Santana, \\ e-mail: joao@uefs.br
}

PALAVRAS-CHAVE: Redes de Sensores sem Fio; Otimização de redes; Mineração de Dados.

\section{INTRODUÇÃO}

O crescente aumento da população em ambientes urbanos tem trazido diversos desafios para os seus habitantes. A preocupação constante em relação a temas como o gerenciamento eficiente de recursos associado com tecnologias de sensoriamento tem motivado iniciativas de pesquisas e desenvolvimento envolvendo o conceito de cidade Cidades Inteligentes (Costa et al. (2017)). Atualmente, as cidades inteligentes são baseadas no contexto de "sistema de sistemas", conjuntura na qual diferentes dispositivos embarcados e inteligentes interagem entre si provendo informações valiosas para a melhoria da qualidade de vida nesses ambientes e, para tanto, operam sobre redes baseadas na Internet ou específicas para a sua aplicação.

A conexão de sensores em forma de uma Rede de Sensores Sem Fio (RSSF) possibilita a criação de infraestruturas distribuídas para monitorar as cidades modernas. Nesse cenário, os sensores são posicionados criteriosamente para que cubram áreas bem definidas, como rodovias, estações de metrô e praças públicas, apenas para citar alguns exemplos.

A natureza das cidades inteligentes, baseada na interação de múltiplos sistemas, traz desafios impactantes e que requerem soluções inovadoras. Um desses desafios se refere ao grande tráfego de informações na rede, uma vez que centenas ou até mesmo milhares de sensores podem estar transmitindo informações simultaneamente. De fato, tal característica requer comunicações com alta vazão e uma alta taxa de confiabilidade na transmissão. Diante desses requerimentos, a otimização da transmissão dos dados coletados possui um caráter extremamente importante no cenário das cidades inteligentes.

Nos últimos anos, os sites de mídias sociais se tornaram bastante populares devido a crescente proliferação de dispositivos capazes de acessar a internet (ex: smartphones, tablets) (Aggarwal (2011)), sendo que essa conclusão pode ser facilmente obtida através da simples análise do número de mídias sociais existentes (ex: Twitter, Facebook, Instagram). Quando os usuários dessas mídias, espontaneamente, postam informações sobre um determinado evento (ex: engarrafamento, assalto), tais eventos podem ser detectados e associados ao seu contexto, a uma região de influência e ao período no qual 
ele ocorreu através de informações explícitas e implícitas nas postagens relativas ao evento (texto, localização e data associados a postagem). Após detectados, os eventos podem ser classificados em uma escala numérica de relevância e associados aos sensores contidos na sua região de influência. Dessa forma, os dispositivos pertencentes aquela área podem otimizar suas tarefas de diversas formas, incluindo a marcação dos pacotes transmitidos ou mudando o comportamento durante a coleta de dados (Costa et al.(2013)). Dentro desse contexto, nós propomos um sistema externo a RSSF a ser utilizado em cidades inteligentes. Tal sistema é responsável por detectar e classificar eventos de interesse para os nós da RSSF, otimizando-a para alta performance.

\section{METODOLOGIA}

O objetivo principal deste trabalho é atribuir níveis de relevância (na forma de índices de prioridade) aos nós sensores de uma RSSF a partir da detecção de eventos de interesse em mídias sociais. O processo completo envolve 3 elementos distintos (Detector de Eventos, Classificador de Eventos e Protocolo de Definição de Prioridades), destacados na Figura 1.

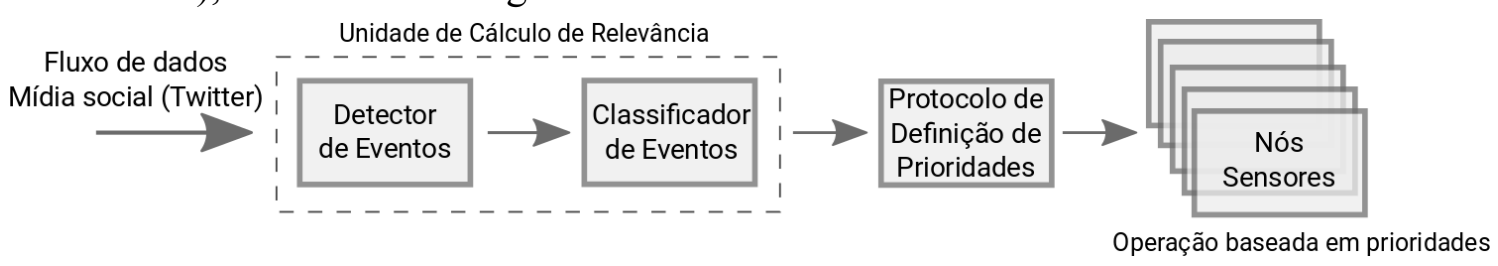

Figura 1: Esquema geral da abordagem proposta

O primeiro módulo do sistema, o Detector de Eventos (DE), faz parte da Unidade de Cálculo de Relevância (UCR) e é responsável por acessar o fluxo de dados de uma mídia social pré-definida em tempo real e a vasculhar procurando por eventos relevantes para a aplicação que, em sequência, são submetidos ao Classificador de Eventos (CE), que estabelece uma única prioridade numérica ao evento detectado. Apesar deste trabalho focar-se no Twitter para extração de dados, outras mídias podem ser utilizadas em trabalhos futuros.

Detector de Eventos (DE): Para obter as especificações do DE, é necessário levar em conta que cidades são um universo que produzem eventos paralelos e incertos. Dentro desse contexto a utilização de DE's que exigem algum nível de supervisão humana se torna impraticável diante da enorme carga de dados que deve ser analisada. Além de operar de forma totalmente automatizada, o DE precisa ser capaz de detectar eventos que estão ocorrendo em regiões geográficas pequenas, como uma praça pública, e possibilitar a extração das características textuais (palavras-chave), espaciais (região impactada) e temporais (janela de tempo na qual o evento está acontecendo). Diante das alternativas disponíveis para a detecção de eventos geo-localizados no Twitter, nós escolhemos o GeoBurst (Zhang et al. (2016)) como base para o DE. Ao agrupar os tweets recebidos em grupos semanticamente coerentes e geograficamente próximos, eventos candidatos são gerados e ranqueados de acordo com o histórico de suas palavras-chave no espaço e no tempo e, consequentemente, eliminando falsos candidatos (como atividades rotineiras, por exemplo). Além dessas características, o 
GeoBurst possui um módulo de atualização que permite a monitoração contínua do fluxo de dados de entrada.

Classificador de Eventos (CE): Uma cidade é suscetível a uma ampla gama de eventos (ex: crimes, alagamentos, engarrafamentos, protestos) e, além de naturezas diferentes, cada evento impacta de uma forma diferente a vida dos cidadãos atingidos por ele. Cada evento que possa vir a acontecer tem um risco (ou prioridade) definido por dois fatores: o escopo e a severidade do evento. Com base na relação escopo-severidade definida por Bostrom (2013), nós estabelecemos quatro níveis de severidade contextual, dispostos na Tabela 1, e criamos um método para calcular o escopo de um evento. Para obter nível de severidade contextual de cada evento, nós construímos um modelo estatístico baseado em amostras de tweets referentes a cada tipo de evento. Ao submeter as palavras-chave de um evento ao modelo, a probabilidade dele pertencer a cada tipo de evento é calculada e a classificação com maior probabilidade é adotada (princípio da máxima a posteriori), resultando no valor da severidade contextual do evento.

Tabela 1. Níveis de severidade contextual.

\section{Severidade contextual}

100

75

50

25
Tipo de Evento

\section{Desastres}

Crimes

Mobilidade

Social

Complementar à severidade textual, o escopo do evento é calculado estimando a população contida na região atingida pelo evento no momento de sua ocorrência. Para tanto, nós consideramos a existência de uma base de dados com uma duração mínima de uma semana e localizada na cidade a ser monitorada e a dividimos em sete grupos diferentes, um para cada dia da semana. Para calcular o escopo, nós executamos uma consulta espacial restringida pela área de impacto do evento no grupo referente ao seu dia da semana e, por fim, calculamos o valor médio dos resultados obtidos.

Protocolo de Definição de Prioridades: A unidade de cálculo de Relevância está completamente operacional, porém o protocolo de definição de prioridades e os algoritmos de configuração dos sensores ainda estão sob desenvolvimento e serão abordadas em trabalhos futuros.

\section{RESULTADOS E DISCUSSÕES}

Nós avaliamos a proposta discutida acima utilizando um dataset real composto por 1.4 milhões de tweets obtido através da API pública de streaming do Twitter. Esses dados representam uma fração de todos os tweets geolocalizados publicados entre 07/02/2017 e 30/04/2017 na cidade de Nova York - EUA. Esse dataset foi processado para verificar os procedimentos de assimilação da prioridade contextual. O algoritmo foi executado em janelas de tempo de uma hora e em sua configuração padrão (Zhang et al (2016)). A Figura 2 demonstra o número médio de cada tipo de evento por dia da semana.

Durante os testes mencionados, a UCR classificou corretamente mais de $70 \%$ dos eventos e, utilizando os resultados obtidos, é possível identificar alguns padrões na Figura 2. Como um exemplo, a quantidade de eventos relativos à mobilidade dobra 
durante os sábados e domingos, indicando que a população enfrenta problemas notáveis relacionados ao tráfego ao decorrer desses dias. Utilizando esses insights, os sistemas da cidade podem ser otimizados para dar suporte a tais eventos, diminuindo o impacto de eventos correntes e de eventos futuros.

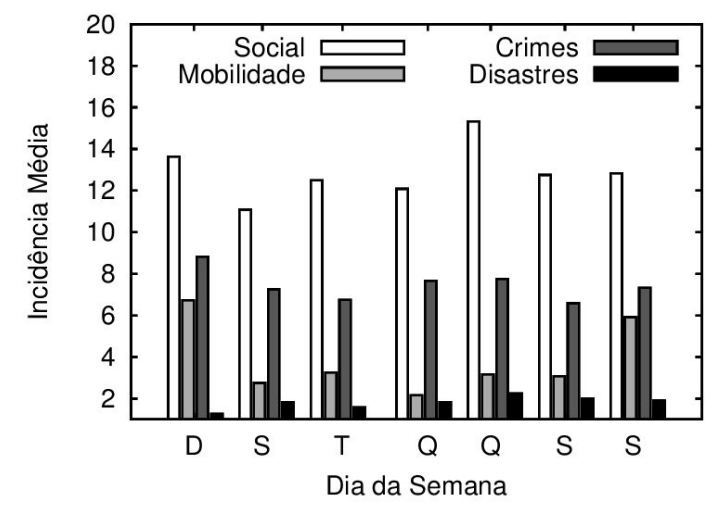

Figura 2: Eventos detectados no dataset de Nova York

\section{CONSIDERAÇÕES FINAIS}

Em uma RSSF, diversos sensores podem ser implantados para recuperar dados escalares (temperatura, pressão, umidade) ou multimídia (som, imagens, vídeos) da área que está sendo monitorada. Diante desse cenário, é natural supor que informações extraídas da Internet possam ser inseridas em RSSF, permitindo diversas estratégias de otimização na rede. Considerando esse cenário e explorando o conceito de crowdsensing, no qual os cidadãos se tornam "sensores" em tempo real da própria cidade, os resultados obtidos mostram que a abordagem proposta pode trazer resultados bastante significativos para o monitoramento de cidades inteligentes utilizando RSSF.

\section{REFERÊNCIAS}

COSTA, D.G.; COLlOTTA, M.; PAU, G. and DURAN-FAUNDEZ, C., 2017. A fuzzy-based approach for sensing, coding and transmission configuration of visual sensors in smart city applications. Sensors, 17(1), p.93.

AGGARWAL, C.C., 2011. An introduction to social network data analytics. Social network data analytics, pp.1-15.

COSTA, D.G.; GUEDES, L.A.; VASQUES, F. and PORTUGAL, P., 2013. Adaptive monitoring relevance in camera networks for critical surveillance applications. International Journal of Distributed Sensor Networks, 9(11), p.836721. ZHANG, C.; ZHOU, G.; YUAN, Q.; ZHUANG, H.; ZHENG, Y.; KAPLAN, L.; WANG, S. and HAN, J., 2016, July. Geoburst: Real-time local event detection in geo-tagged tweet streams. In Proceedings of the 39th International ACM SIGIR conference on Research and Development in Information Retrieval (pp. 513-522). ACM.

BOSTROM, N., 2013. Existential risk prevention as global priority. Global Policy, 4(1), pp.15-31. 